\title{
Pathogenesis of chlamydial polyarthritis in domestic animals: characteristics of causative agent
}

\author{
J. STORZ AND P. SPEARS \\ From the Colorado State University, Fort Collins, and the National Jewish Hospital, Denver, Colorado, USA
}

Since chlamydial agents were first recognised as a cause of polyarthritis in lambs ${ }^{222}, 302$ they have been found to cause similar diseases in calves ${ }^{188,}{ }^{305}$ and swine. ${ }^{187}$ Chlamydial infections leading to polyarthritis in lambs and calves and the nature of the causative agent have been extensively investigated. ${ }^{75}, 222,289,303,304$ We shall analyse in this report the properties that distinguish chlamydial strains with arthropathogenic potential and the critical events in the pathogenesis of this infection.

\section{Differentiation of arthropathogenic chlamydiae}

The strains of Chlamydia psittaci isolated from joints, conjunctiva, blood, visceral organs, or intestines of lambs and calves affected with polyarthritis have antigenic and biological properties that distinguish them from other $C$. psittaci strains. ${ }^{299,} 300$ Antigenic differences have been demonstrated between ovine and bovine chlamydial strains that cause abortions and those that cause polyarthritis. ${ }^{270,271,} 299$ Using strain-specific serum neutralisation testing, it was seen that all polyarthritis-producing Chlamydiae, whether from cattle or sheep, share an antigen which allows these chlamydial strains to be placed into serotype 2 . Serotype 2 strains have been isolated only from animals affected with polyarthritis or conjunctivitis. Strains of $C$. psittaci isolated from cattle and sheep with clinically inapparent intestinal infections or abortions were placed into serotype 1 .

When mouse $\mathrm{L}$ cells were infected with serotype 2 strains and stained by the Giemsa method inclusions were detected after 18 hours as irregular patches of reticulate bodies in the perinuclear region. The inclusions developed outward from this region to form compact areas of chlamydial forms and lobed or diffuse areas in which the chlamydiae developed interspersed with host cytoplasm. The inclusion membrane separated the pleomorphic inclusion of the prokaryotic parasite from the eukaryiotic cytoplasm at all times. When a young monolayer with dividing cells was infected with a serotype 2 strain, so that a high percentage of the cells developed inclusions, many infected dikaryons were formed early in the infection. The ability to induce dikaryons has been observed only with the serotype 2 strains and represents a unique effect on the cytoskeletal apparatus of the host cell. Furthermore, the strains altered the cytoskeleton of all infected cells late in the developmental cycle, causing host cells to round up as the inclusions matured. ${ }^{293}$

All Chlamydiae are relatively inefficient in infecting cultured cells in monolayers. ${ }^{306}$ Several methods have been used to promote infectivity of serotype 2 strains. The most effective method was centrifugation of chlamydial elementary bodies on to the host cells. The $\mathrm{L}$ cell infectious units of a yolk-sac-propagated serotype 2 strain were 75 times higher when the titre was measured by centrifugation instead of stationary adsorption. The lag time before inclusions were visible was longer in centrifuged cells. Inclusions induced by serotype 2 strains were not large enough to be counted until after 40 hours, whereas inclusions in uncentrifuged monolayers could be counted earlier. The significance of the longer lag time is not known.

Another method that is often used to enhance chlamydial infectivity is to treat cell monolayers with the polycation DEAE-dextran (DEAE-D) before adding the inoculum. ${ }^{149}$ An average of nine times the number of cells were infected by serotype 2 strains in DEAE-D-treated compared with untreated monolayers. ${ }^{293}$ However, the yield of infectious progeny per infected cell from DEAE-D-treated monolayers was smaller. In contrast, DEAE-D had little effect on the number of inclusions formed or the yields of a serotype 1 abortion strain of $C$. psittaci. The ability of serotype 2 strains to form inclusions in almost 10 times more cells of monolayers treated with DEAE-D is an important biological marker for these chlamydiae (Table).

Cycloheximide has also been used to enhance development of several types of $C$. psittaci, probably by depressing protein synthesis of the eukaryotic host cell to provide the chlamydiae the use of a larger 
Table Response of Chlamydia psittaci serotypes 1 and 2 to DEAE-dextran $(D D)$ and cycloheximide $(\mathrm{CH})$. Count in treated cells/count in untreated cells

\begin{tabular}{|c|c|c|c|}
\hline & DD & $\mathrm{CH}$ & $\mathrm{DD}+\mathbf{C H}$ \\
\hline $\begin{array}{l}\text { Serotype 1 } \\
\text { Inclusions } \\
\text { Yields }\end{array}$ & $\begin{array}{l}1 \cdot 1(0 \cdot 8-1 \cdot 7)^{*} \\
1 \cdot 1\end{array}$ & $31^{3 \cdot 0(2 \cdot 7-3 \cdot 4)}$ & $4^{3 \cdot 8(2 \cdot 6-4 \cdot 7)}$ \\
\hline $\begin{array}{l}\text { Serotype } 2 \\
\text { Inclusions } \\
\text { Yields }\end{array}$ & $\begin{array}{l}9 \cdot 3(4 \cdot 9-14)^{*} \\
1.9\end{array}$ & $\begin{array}{l}1 \cdot 6(1 \cdot 2-2 \cdot 6) \\
0 \cdot 2\end{array}$ & $\begin{array}{l}10 \cdot 9(6 \cdot 2-17) \\
0.3\end{array}$ \\
\hline
\end{tabular}

*Range of values for 4 strains.

soluble amino-acid pool. ${ }^{151}$ Development of serotype 2 strains was depressed in cycloheximide-treated cells. Although these strains initiated about $50 \%$ more inclusions in the presence of cycloheximide, the yield of infectious progeny was only $20 \%$ of that from untreated cells. In comparison, an abortion strain of $C$. psittaci yielded 10 times more infectious progeny when cycloheximide was added to the culture medium. Ultrastructural studies of a serotype 2 strain in cells treated with cycloheximide revealed pycnotic chlamydial forms in many inclusions. The synergistic actions of inhibitor and parasite damaged mitochondria of the host cell. These findings indicated that the serotype 2 strains are more dependent on host cell functions suppressed by cycloheximide than are other $C$. psittaci strains tested (Table).

\section{Experimental induction of polyarthritis by serotype 2 strains}

Chlamydial agents have been isolated directly from the sites of the major lesions, the affected joints of naturally occurring cases of polyarthritis in lambs and calves. In addition to the synovial fluid and tissue samples specimens from lungs, liver, spleen, kidneys, brain, lymph nodes, conjunctiva, and from different intestinal levels yielded chlamydial agents by yolk-sac cultivation after inoculating 7-day-old chicken embryos. ${ }^{222,302,305}$ The spontaneous disease picture of polyarthritis could readily be reproduced experimentally by inoculating lambs or calves via the oral, subcutaneous, intramuscular, intravenous, or intra-articular routes with yolk-sac propagated chlamydiae of serotype $2 .{ }^{75}, 222,239,303,304$ Animals inoculated with chlamydiae of serotype 1 did not develop joint infection, and this serotype was never isolated from joint specimens or conjunctival scrapings. ${ }^{299,} 300$

Three days after parenteral inoculation of lambs serotype 2 chlamydiae could be isolated from specimens of several joints, although gross lesions were minimal. Hip, knee, shoulder, and elbow joints of lambs examined seven days after had moderate to severe inflammatory changes, and chlamydiae were isolated from these sites. Involvement of most joints of the experimental lambs was evident at 21 days. Chlamydiae were isolated from affected joints at this time. Twenty-eight days after inoculation, 239,303 however, cultures of synovial fluids were all negative for infectious agents.

\section{Significance of intestinal phase of infection}

Oral exposure is an important route of infection under natural conditions. ${ }^{300}$ Intestinal infection and penetration of the intestinal mucosa was studied in calves after oral inoculation with a serotype 2 strain. ${ }^{85,86}$ Infection and multiplication occurred in the mucosa of the abomasum and the small and large intestine, causing primary diarrhoea. Newborn calves often died from this primary diarrhoea. Calves 7 days old at the time of inoculation survived the primary diarrhoea and developed polyarthritis and polyserositis. Chlamydiae were recovered from the blood and the affected synovial tissues 4 to 25 days after oral inoculation.

Fluorescent antibody tracings and electron microscopic studies revealed that intestinal epithelial cells on the tips of the villi and on the intervillous zones were infected, but a few cells in the crypts of Lieberkühn and the transition zones also contained chlamydial inclusions. Leucocytes and lymphocytes were attracted by intestinal cells containing chlamydial inclusions. The following cell types in decreasing order of frequency contained chlamydiae at different stages of development: absorptive epithelial cells, macrophages and fibroblasts in the lamina propria, endothelial cells in the central lacteals, enterochromaffin and goblet cells of the villi, and undifferentiated cells in the crypts of Lieberkühn. The chlamydial infection of these cells was cytocidal. The infected endothelial cells burst to release chlamydiae into the lymphatic and blood circulation. This marked the beginning of the chlamydaemic phase during which infection of the synovial target tissue occurred.

\section{Changes in chlamydia-infected synovial tissues}

The most striking tissue changes were articular and periarticular in both field and experimental cases in lambs and calves. ${ }^{75,}$ 289, 303 Periarticular hyperaemia and oedema were associated with pronounced intra-articular lesions. Affected joints contained excessive amounts of greyish-yellow, turbid synovia 
fluid and plaques of fibrin. The tendon sheaths were distended and contained turbid synovial fluid. Surrounding muscles were hyperaemic and oedematous with petechiae on the associated fascial planes.

The reactive histological changes in synovial membranes, joint capsules, tendon sheaths, tendons, ligaments, periarticular connective tissue, and muscles were inflammatory and proliferative (Figs 1-6). Synovial lining cells became swollen and hyperplastic, and their size varied. As the infection progressed, the synovial lining cells assumed a pseudostratified appearance. Infiltration with leucocytes and lymphocytes occurred at this stage. The synovial connective tissue became hyperaemic and oedematous, and foci of mononuclear cells accumulated. In advanced stages the joint capsules and tendon sheaths thickened fibrotically. This was associated with an extensive inflammatory response that consisted primarily of infiltration by monocytes, lymphocytes, macrophages, and plasma cells. Electron microscopic investigations on infected synovial tissues were reported, but the stages of chlamydial development were not related sufficiently to the cellular changes in the synovial tissues.

In cultured cells maturation of chlamydial inclusions caused activation of the lysosomes. ${ }^{314}$ Subsequent lysis of the host cell released lysosomal enzymes and cell debris. These released enzymes could contribute to the inflammatory reaction and damage to synovial tissue in the course of the infection.

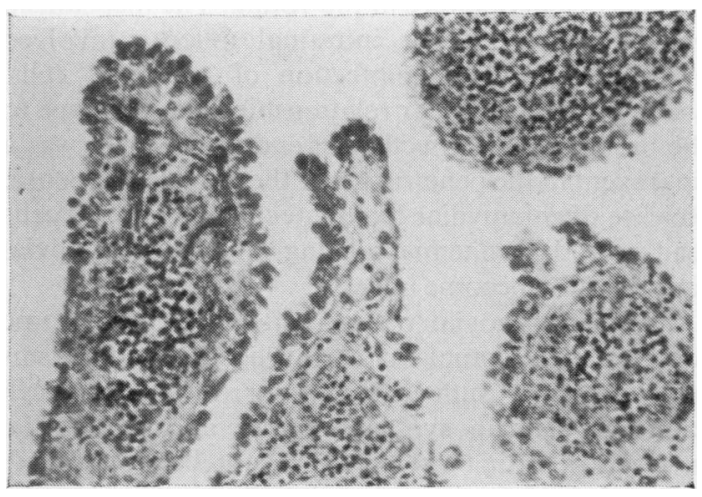

Fig. 1 Villi of synovial membrane of lamb with naturally occurring chlamydial polyarthritis. Note pseudostratified synovial cells, hyperaemia, and infiltration of lymphoblastoid cells. (Haematoxylin and eosin).

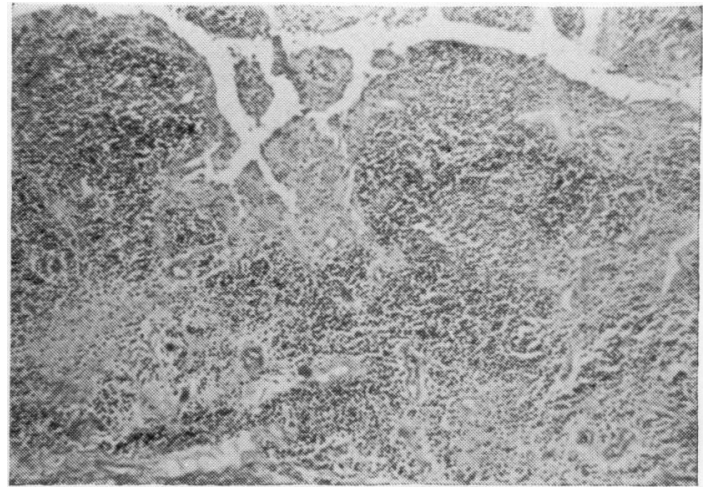

Fig. 2 Synovial membrane with severe inflammatory reaction in a polyarthritic lamb with chlamydial infection. (Haematoxylin and eosin.)

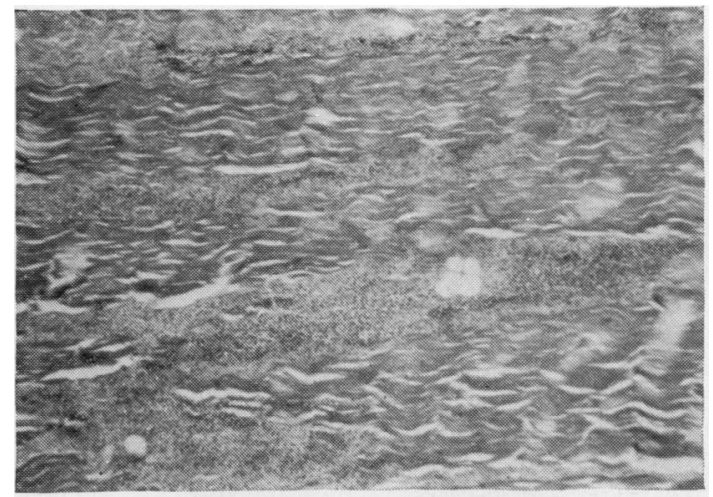

Fig. 3 Inflammatory reaction in skeletal muscle from a polyarthritic lamb. Note inflammatory cells and oedema between muscle fibres. (Haematoxylin and eosin.)

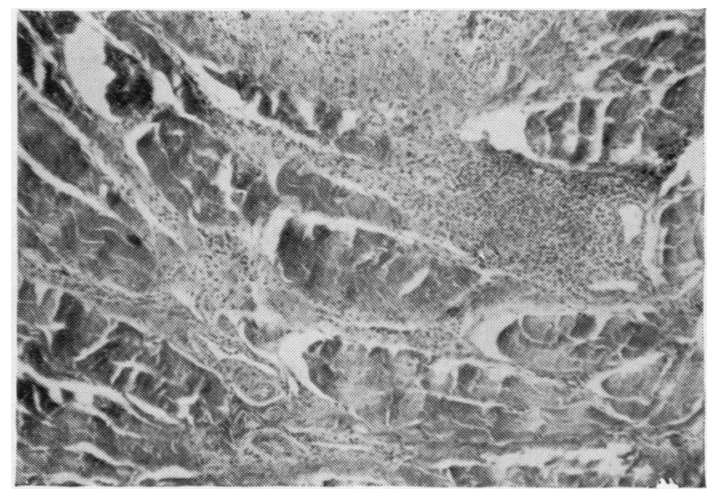

Fig. 4 Affected tendon with accumulation of inflammatory cells and oedema. (Haematoxylin and eosin). 


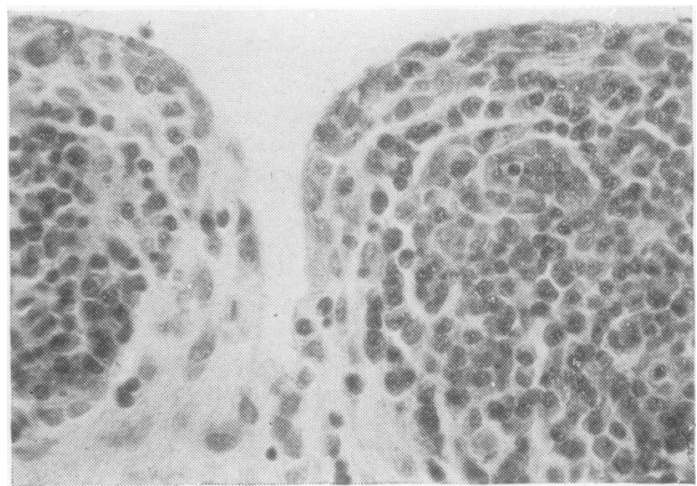

Fig. 5 Granulomatous infiltrations of pars synovialis with various types of inflammatory cells 21 days after intramuscular inoculation of lamb with type 2 chlamydial strain. (Haematoxylin and eosin.)

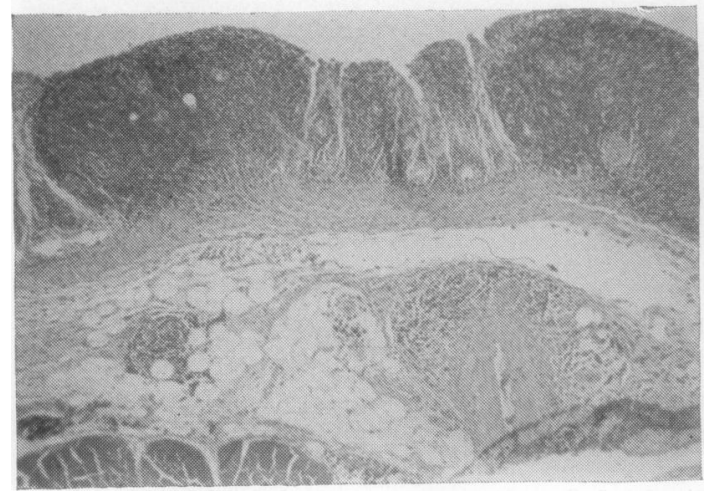

Fig. 6 Tendovaginitis and peritendinitis 21 days after inoculation of lamb with type 2 chlamydial strain. (Haematoxylin and eosin.)

\section{Conjunctival and genital lesions}

Chlamydiae of serotype 2 were isolated from lambs with keratoconjunctivitis who did not have joint involvement. However, virtually all lambs with polyarthritis also had conjunctivitis. Chlamydial cytoplasmic inclusions were detected in conjunctival cells of these lambs. ${ }^{297,} 300$

Sexually mature rams that were inoculated parenterally developed chlamydaemia and polyarthritis. About five to seven days after inoculation, when chlamydaemia ceased, they excreted chlamydiae in semen and continued to do so for extended periods. The semen quality deteriorated and became purulent. ${ }^{301}$ chlamydiae were isolated from testicles, epididymis, and from the accessory sex glands.

Some of the calves developed serositis of peritoneal and pleural cavity surfaces after oral or parenteral inoculation. The visceral organs were then covered with a thin layer of fibrinous frosting.

\section{Discussion and conclusions}

Chlamydial agents of serotype 2 were isolated from lambs and calves affected with polyarthritis, conjunctivitis, and intestinal infections. Disease identical to the naturally occurring one was induced by subcutaneous, intramuscular, intravenous, intraarticular, or oral exposure. These investigations identified chlamydiae of serotype 2 as a unique and important pathogen that has specific arthropathogenic properties which were not detected in chlamydiae associated with other disease conditions of cattle and sheep. This agent also induced polyarthritis in dogs, but it has not been tested in other animal species. Chlamydiae isolated from joints of arthritic swine have not been sufficiently characterised, and comparative investigations are needed. ${ }^{187}$ The chlamydial infections described present unique models to study the pathogenesis of infections leading to polyarthritis.

Intestinal infection was recognised as an important initial step in the pathogenesis of chlamydial polyarthritis. This infection was cytocidal for a wide range of different intestinal epithelial cells as well as macrophages and endothelial cells. The loss of cell functions and the accelerated cell death induced increased cell turnover and primary diarrhoea that was particularly severe in the young. The mechanism of penetration of the intestinal mucosa involved cytocidal chlamydial infection of epithelial cells, changes in intercellular relationships, and damage to the basal lamina. Infection of endothelial cells was a final event in the penetration of the intestinal mucosa. Release of chlamydiae from infected endothelial cells lead to chlamydaemia, during which the synovial target organs become infected.

Different synovial tissues and structures became infected. The actual arthropathogenic mechanisms have not been sufficiently explored. The host cell range of cells in synovial tissues and chlamydial interaction with these cells has not been explored. Infected intestinal epithelial cells attracted polymorphonuclear leucocytes and lymphocytes. Chlamydia-cell interactions in synovial tissues and the ultimate effect of chlamydial multiplication on these cells need to be explored.

Unique properties were detected in chlamydiae of 
serotype 2 . Only strains with this antigenic make-up were isolated from animals affected with polyarthritis. Furthermore, only these chlamydial strains had arthropathogenic properties. The response of serotype 2 strains to host cells treated with cycloheximide or DEAE-dextran further distinguish these strains. An important implication of the cycloheximide effect is that these strains are more dependent on cellular functions than other strains of $C$. psittaci or $C$. trachomatis. This high degree of dependence on eukaryotic host cell functions may contribute to the unique pathogenic potential of the chlamydial strains causing polyarthritis.

\section{General discussion}

PROF. A. S. RUSSELL: If you can show the organisms in semen have you documented venereal transmission too?

PROF. STORZ: Initially the disease does not have to be transmitted sexually. Once an animal is infected and excretes chlamydia in the semen it becomes temporarily sterile. But not so if normal semen was contaminated in vitro and inseminated artificially. After fertilisation from a diseased bull or ram we could occasionally harvest embryos that had reached the eight to sixteen cell stage, but the intrauterine environment was probably changed and nidation or pregnancy did not occur. Infection of the endometrium has been observed but no other adverse effects.

PROF. A. E. GOOD: Was it your opinion that diverse disease manifestations were due to infection in the various organs rather than to a 'reactive process'?
PROZ. STORZ: On the one hand, we traced the infection in the joints of lambs and calves for 21 to 28 days. On the other hand, we did electron microscope studies with Dr. Walter Norton and found that the 'reactive process', as you call it, persisted for quite some time beyond the infectious stage. But even in that reactive stage we saw unusual inclusions and were not sure whether they were chlamydial in nature.

PROF. M. ZIFF: There were very few plasma cells in your synovial inflammation. This looks like a cellular rather than a humoral immune response.

PROF. STORZ: We studied the relationship of the infectious agent with the different cells. The intestinal tract macrophages in the young animal were readily infected and were good substrates for chlamydial replication. Plasma cells also were taking up the agent but the chlamydial multiplication process was arrested. At 56 days the joint lesions contained plasma cells.

DR. E. ALBERT: Concerning the susceptibility to this infection, did you have any animals that did not get infected with a standard oral dose of chlamydia? If so, was there a sex difference?

PROF. STORZ: We did not look at the sex difference. We just took calves as we got them. Susceptibility varies with age. Younger animals are much more susceptible to oral exposure. We could infect almost all lambs that were free of chlamydial antibodies and induce arthritis. Sometimes the reaction was not quite so severe. When they were left alone and fed, so that they would not have to walk, the reaction was less severe. Rest helps them as it does humans with arthritis. 